\title{
EFICIÊNCIA DOS GASTOS EM EDUCAÇÃO TÉCNICA NO BRASIL: ANÁLISE DOS IFs - INSTITUTOS FEDERAIS
}

\author{
EFFICIENCY OF EXPENSES IN TECHNICAL EDUCATION IN \\ BRAZIL: ANALYSIS OF THE FI - FEDERAL INSTITUTES
}

\section{EFICIENCIA DEL GASTO EN EDUCACIÓN TÉCNICA EN BRASIL: ANÁLISIS DE FI - FEDERAL INSTITUTES}

\author{
Oderson Panosso ${ }^{1}$ \\ Gleice Carvalho de Lima Moreno ${ }^{2}$ \\ Nelson $\mathrm{Hein}^{3}$ \\ Adriana Kroenke Hein ${ }^{4}$ \\ Artigo recebido em novembro de 2020 \\ Artigo aceito em julho de 2021
}

\begin{abstract}
RESUMO
Os IFs - Institutos Federais do Brasil - foram criados em com objetivo de formação técnica no Brasil. Atualmente os IFs possuem 38 unidades que fazem parte da estrutura cumprindo os objetivos de atingir o máximo de alunos possíveis espalhados pelos interiores do país. O objetivo deste estudo é comparar a eficiência dos investimentos das unidades do IFs no País fazendo uma comparação com a eficiência acadêmica definida nos Plano Nacional da Educação. Para alcançar este objetivo foi realizada uma pesquisa descritiva, documental e quantitativa, com extração de dados da Plataforma Nilo Peçanha, com tratamento e análise dos dados pelo método multicritério de análise envoltória de dados (DEA) a partir do modelo BCC com orientação para Output. O ano analisado foi 2018. O resultado obtido se observou que $47 \%$ das IFs são consideradas eficientes. Entre as unidades consideradas eficientes estão os IFs o IFNMG, o IFPA, o IFRO, IFBA, IFCE, IFRN, IFAP e IFRR, IFPI e IFMS, IFMT e IFPR e IFMA. Notou-se que nem todos os IFs que foram considerados eficientes tecnicamente estão classificados como Eficientes Acadêmicos, ou seja, os IFs precisam melhorar a Eficiência Acadêmica para alcançar os objetivos do Plano Nacional da Educação justificando os investimentos aplicados. As demais unidades consideradas ineficientes merecem uma atenção para um controle maior da aplicação dos recursos.
\end{abstract}

Palavras-chave: Eficiência. Investimentos. Educação. Análise envoltória de dados (DEA).

\footnotetext{
1 Doutorando em Contabilidade pelo IFRS - Instituto Federal do Rio Grande do Sul. E-mail: opanosso@gmail.com.

${ }^{2}$ Doutoranda em Contabilidade pela UNIR - Universidade Federal de Rondônia. E-mail: gleicemoreno@ unir.br.

${ }^{3}$ Doutor em Engenharia da Produção pela FURB - Universidade Regional de Blumenau. E-mail: hein@ furb.br.

${ }^{4}$ Doutora em Métodos Numéricos e Engenharia pela FURB - Universidade Regional de Blumenau. E-mail: akroenke@ furb.br.
}

Refas - ISSN 2359-182X v.7, n.6

Agosto de 2021 


\begin{abstract}
The IFs - Instituto Federais do Brasil were created in order to provide technical training in Brazil. Currently the IFs have 38 units that are part of the structure, fulfilling the objectives of reaching the maximum number of possible students spread across the interiors of the country. The purpose of this study is to compare the investment efficiency of the IFs units in the country by making a comparison with the academic efficiency defined in the National Education Plan. To achieve this objective, a descriptive, documentary and quantitative research was carried out, with data extraction from the Nilo Peçanha Platform, with treatment and analysis of the data by the multicriteria method of data envelopment analysis (DEA) using the BCC model with Output orientation. The year analyzed was 2018. The result obtained was observed that $47 \%$ of the IFs are considered efficient. Among the units considered efficient are the IFs, the IFNMG, the IFPA, the IFRO, IFBA, IFCE, IFRN, IFAP and IFRR, IFPI and IFMS, IFMT and IFPR and IFMA. It was noted that not all IFs that were considered technically efficient, are classified as Academic Efficient, that is, IFs need to improve Academic Efficiency to achieve the objectives of the National Education Plan justifying the investments applied. The other units considered inefficient deserve attention for greater control over the use of resources.
\end{abstract}

Keywords: Efficiency. Investments. Education. Data envelopment analysis (DEA).

\title{
RESUMEN
}

Las FI - Instituto Federais do Brasil se crearon con el fin de brindar capacitación técnica en Brasil. Actualmente las FI cuentan con 38 unidades que forman parte de la estructura, cumpliendo los objetivos de llegar al máximo número de posibles alumnos repartidos por el interior del país. El propósito de este estudio es comparar la eficiencia de inversión de las unidades de la FI en el país mediante una comparación con la eficiencia académica definida en el Plan Nacional de Educación. Para lograr este objetivo se realizó una investigación descriptiva, documental y cuantitativa, con extracción de datos de la Plataforma Nilo Peçanha, con tratamiento y análisis de los datos por el método multicriterio de análisis envolvente de datos (DEA) utilizando el modelo BCC con orientación Output. El año analizado fue 2018. El resultado obtenido se observó que el 47\% de las FI se consideran eficientes. Entre las unidades consideradas eficientes se encuentran las FI, IFNMG, IFPA, IFRO, IFBA, IFCE, IFRN, IFAP e IFRR, IFPI e IFMS, IFMT e IFPR e IFMA. Se observó que no todas las FI que se consideraron técnicamente eficientes, se clasifican como Eficientes Académicamente, es decir, si necesitan mejorar la Eficiencia Académica para lograr los objetivos del Plan Nacional de Educación justificando las inversiones aplicadas. Las otras unidades consideradas ineficientes merecen atención para un mayor control sobre el uso de los recursos.

Palabras Clave: Eficiencia. Inversiones. Educación. Análisis envolvente de datos (DEA).

\section{INTRODUÇÃO}

O cenário da educação no ensino técnico no Brasil passou por fortes mudanças desde a criação e consolidação dos Institutos Federais espalhados pelo Brasil. Esta oferta de vagas no interior do país vem mudando a forma de desenvolvimento do País. O Instituto Federal de Educação, Ciência e Tecnologia (IF) foi criado em 29 de dezembro de 2008, pela lei 11.892, que instituiu, no âmbito do sistema federal de ensino, a Rede Federal de Educação Profissional, Científica e Tecnológica, vinculada ao Ministério da Educação e constituída por 38 Institutos Federais de Educação, Ciência e Tecnologia espalhados pelo país. Essas instituições possuem autonomia administrativa, patrimonial, financeira, didático-pedagógica e disciplinar. 
As unidades IFs tem sua autonomia para atender a localidade oferecendo cursos que ajudem no desenvolvimento da região instalada. Logo a forma de gestão mais dinâmica e uma estrutura organizacional mais flexível pode se demonstrar mais eficiente que as demais unidades. As unidades podem ser diferentes quanto as característica internas, obedecendo a mesma legislação, o que pode influenciar em eficiência maior quando comparadas as demais unidades quanto aos seus próprios indicadores.

No ano de 2018 foram gastos mais de 14 bilhões de reais para manutenção com os IFs. Portanto se torna importante buscar analisar a eficiência de suas unidades para melhor entendimento do uso de recursos públicos em educação no país.

Alguns estudos tem buscado analisar a eficiência no IFs (Instituo Federais), como o estudo de Rodrigues, Muylder e Gontijo (2018), que avaliaram eficiência de nove unidades do Centro Federal de Educação Tecnológica de Minas Gerais em 2015 e verificou que apenas duas unidades foram globalmente eficientes. Já no trabalho de Krieser et al. (2017) objetivou identificar a eficiência técnica dos IFs no Brasil utilizando o método DEA. Foi analisada que as unidades no Brasil demonstrando um ranking identificando os que servem de benchmark, que totalizaram 47,36\% (09 DMUs). Logo, a função de analisar a eficiência em instituições de ensino pode trazer resultados sobre a capacidade das unidades em relação aos seus recursos.

Analisar a eficiência para Belloni (2000) está relacionado à capacidade produtiva que está relacionado com a habilidade de evitar desperdícios. Ou seja, voltada para elevar níveis de produção mantendo as quantidades de insumos inalteradas ou de forma equivalente mantendo níveis de produtos invariáveis reduzindo a quantidade de insumos no processo de produção. E ainda segundo Belloni (2000) a eficiência está relacionada à forma de alocação da capacidade de combinar de forma ótima, insumos e produtos dados os preços existentes.

Peña (2008) conceitua eficiência como a combinação ótima dos insumos e métodos necessários, (inputs) no processo produtivo de modo que gerem o máximo de produto (output), ou seja, eficiência é a capacidade de fazer adequadamente as coisas, de minimizar a relação insumos - produtos.

Entre os métodos mais utilizados para medir a eficiência do setor educacional destacase a análise envoltória de dados (DEA) data envelopment analysis, em inglês - que utiliza das técnicas de programação matemática e permite o cômputo de múltiplos insumos e produtos, característica da produção de ensino. Além disso, a DEA não exige uma forma funcional da função de produção, o que elimina o problema de má-especificação do modelo. (COSTA et al., 2015)

Portanto, como problema de pesquisa busca se responder: qual a eficiência das 38 unidades do IFs - Instituo Federais do Brasil sob o sistema DEA? Para responder esta pergunta, o objetivo do artigo é analisar a eficiência das 38 unidades do IFs espalhadas pelo Brasil sob o sistema DEA.

Além desta introdução, este artigo contém mais quatro sessões. A segunda apresenta o IFRS e estudos semelhantes. Na terceira, são detalhados os métodos de pesquisa. Na seção quatro, discutem-se os resultados encontrados, e, por fim, têm-se as considerações finais. 


\section{REFERENCIAL TEÓRICO}

Inicia-se o referencial teórico pelos IFs.

\subsection{IF - Instituto Federal do Brasil}

Integrante do sistema federal de ensino vinculado ao Ministério da Educação, a Rede Federal foi instituída pela reunião de um conjunto de instituições: Institutos Federais de Educação, Ciência e Tecnologia (Institutos Federais); Universidade Tecnológica Federal do Paraná - UTFPR; Centros Federais de Educação Tecnológica Celso Suckow da Fonseca do Rio de Janeiro (Cefet-RJ) e de Minas Gerais (Cefet-MG); Escolas Técnicas vinculadas às Universidades Federais; e Colégio Pedro I.

Em 2019, a Rede Federal está composta por 38 Institutos Federais, 02 Centros Federais de Educação Tecnológica (Cefet), a Universidade Tecnológica Federal do Paraná (UTFPR), 22 escolas técnicas vinculadas às universidades federais e o Colégio Pedro II.

Considerando os respectivos campi associados a estas instituições federais, têm-se ao todo 661 unidades distribuídas entre as 27 unidades federadas do país.

Essas instituições possuem autonomia administrativa, patrimonial, financeira, didático-pedagógica e disciplinar.

No âmbito do Ministério da Educação, compete à Secretaria de Educação Profissional e Tecnológica (Setec/MEC) o planejamento e o desenvolvimento da Rede Federal de Educação Profissional, Científica e Tecnológica, incluindo a garantia de adequada disponibilidade orçamentária e financeira.

Logo, este trabalho focou nos 38 Institutos federais espalhados em vários pontos no Brasil.

\subsection{Estudos Anteriores}

Silva, Corrêa e Gomes (2017) afirma que eficiência é a otimização de recursos e a ausência de desperdício, ou seja, a busca pela melhoria e pelo aumento qualitativo e quantitativo da produção, com alocação eficiente de insumos. Eficiência é a combinação ótima dos insumos e métodos necessários (inputs) no processo produtivo de modo que gerem o máximo de produto (output) (KUAH e WONG, 2011; AOKI et al. 2010, PEÑA, 2008).

Eficiência pode ser percebida como a habilidade de fazer o uso mais adequado do que se tem à disposição, a fim de alcançar um resultado pretendido, podendo ser considerada uma medida da capacidade que agentes ou mecanismos têm para melhor atingir seus objetivos, produzindo o efeito por eles esperado, em função dos recursos disponíveis. (WORTHINGTON, 2001).

Logo a avaliação da eficiência de Instituições de Educação Profissional, Científica e Tecnológica foi o propósito dos estudos desenvolvidos por Ramos e Ferreira (2007), que realizou uma aplicação de Análise de Envoltória de Dados - DEA para avaliar a existência de retornos constantes ou variáveis de escala, no desempenho de instituições de ensino tecnológico no Brasil, com ênfase no ensino médio. Os principais resultados sugerem haver retornos variáveis de escala para orçamento e corpo docente, mas retorno constante para titulação. 
Para o estudo de Almeida e Almeida Filho (2014), o objetivo foi avaliar o nível de eficiência técnica da gestão das escolas federais de educação básica no Brasil, tendo em vista identificar as escolas com a melhor combinação de insumos e resultados educacionais. Os resultados centrais desta pesquisa apontam que as escolas federais situadas no estado do Espírito Santo apresentam, em média, os maiores índices de eficiência técnica.

No artigo de Furtado e Campos (2015), o objetivo foi identificar a escala de eficiência técnica dos Institutos Federais de Educação, Ciência e Tecnologia e a relação de custo, indicativa de expansão e retenção nos escores de eficiência, compreendendo o período de 2012 e 2013, compondo uma amostra formada por 19 unidades. O resultado demonstrou eficiência em apenas seis dos institutos analisados alcançaram a pontuação eficiência em 2012 e 2013. As evidências de os institutos considerados eficientes apresentaram melhor resultados médios de estudantes conclusivos matriculados e despesas correntes mais baixas por alunos matriculados, indicando que a obtenção do resultado não está condicionada a despesa.

No estudo de Rodrigues, Muylder e Gontijo (2018) o foco foi avaliar a eficiência de nove unidades do Centro Federal de Educação Tecnológica de Minas Gerais em 2015, utilizando dos modelos de Data Envelopment Analysis. Os resultados apresentaram apenas duas unidades do Centro Federal de Educação Tecnológica de Minas Gerais foram globalmente eficientes.

Santos (2018) teve como objetivou analisar de forma comparativa a eficiência dos campi do Instituto Federal de Farroupilha baseado em aspectos evolutivos no período entre 2010 e 2016 em nove unidades. Entre os resultados apurou-se que apenas duas unidades do Centro Federal de Educação Tecnológica de Minas Gerais foram globalmente eficientes.

Portanto, esses estudos ainda não apresentaram uma analise da eficiência dos IFs referente ao ano de 2018 considerando os dados de todos os gastos e estrutura em relação aos resultados.

\section{MÉTODO}

Está é uma pesquisa quantitativa, exploratória e descritiva com dados secundários obtidos na plataforma Nilo Peçanha, referentes ao ano de 2018. O tratamento destes dados ocorreu pela análise multicritério de dados a partir do método de análise envoltória de dados (Data Envelopment Analysis - DEA).

A amostra da pesquisa compreendeu 38 unidades que compõem o IFs atualmente.

Quadro 1 - Institutos Federais do Brasil

\begin{tabular}{|c|c|c|c|}
\hline \multicolumn{4}{|c|}{ Localidade de Ensino } \\
\hline DMU & Tipo & DMU & Tipo \\
\hline UN1 & IFAC & UN20 & IFPA \\
\hline UN2 & IFAL & UN21 & IFPB \\
\hline UN3 & IFAM & UN22 & IFPI \\
\hline UN4 & IFAP & UN23 & AgOsto de 2021 \\
\hline
\end{tabular}




\begin{tabular}{|c|c|c|c|}
\hline UN5 & IFB & UN24 & IFPR \\
\hline UN6 & IFBA & UN25 & IFRJ \\
\hline UN7 & IFBAIANO & UN26 & IFRN \\
\hline UN8 & IFC & UN27 & IFRR \\
\hline UN9 & IFCE & UN28 & IFRS \\
\hline UN10 & IFES & UN29 & IFS \\
\hline UN11 & IFF & UN30 & IFSC \\
\hline UN12 & IFARROUPILHA & UN31 & IFSP \\
\hline UN13 & IFG & UN32 & IFSUDESTE \\
\hline UN14 & IFGOIANO & UN33 & IFSUL \\
\hline UN15 & IFMA & UN34 & UN35 \\
\hline UN16 & IFMG & UN36 & IFSULDEMINAS \\
\hline UN17 & IFMS & UN37 & IFTM \\
\hline UN18 & IFMT & UN38 & IFTO \\
\hline UN19 & IFNMG &
\end{tabular}

Fonte: Dados da pesquisa

O Quadro 1 apresentada todas as unidades que compõem o IFs atualmente. A sigla UN é utilizada para identificação de Unidade.

\subsection{Definição de DEA}

Análise Envoltória de Dados é um método quantitativo (Data EnvelopmentAnalysis DEA), cujos precursores foram Koopmans (1951), Debreu (1951) e Farrel (1957), que utilizaram técnicas não paramétricas para avaliar a eficiência.

Esse método fornece uma comparação entre unidades através de dados quantitativos sobre possíveis direções do desempenho, o qual consiste em uma técnica multivariada não paramétrica que analisa a produtividade (ou eficiência) de Unidades Tomadoras de Decisão (Decision Making Units - DMUs). (GIACOMELLO e OLIVEIRA, 2014).

Existem dois modelos de DEA, o modelo Constant Returns to Scale, desenvolvido por Charnes, Cooper e Rhodes em 1978 (CCR), que apresenta retornos constantes de escala e o modelo criado por Banker, Charnes e Cooper em 1998 (BCC), desenvolvido para acomodar efeitos de distinção entre ineficiências técnicas e de escala em sua análise (TAVARES e MEZA, 2017).

Em estudos de anteriores (Santos, 2018; Rodrigues, Muylder e Gontijo, 2018; Furtado e Campos, 2015), foram avaliados pelo sistema DEA com características diferentes. Logo este sistema pode contribuir com a análise dos IFs.

A Figura 1 ilustra o formato das fronteiras nos dois modelos clássicos de DEA. Dessa forma, é possível perceber que no modelo de retornos constantes de escala as DMUs "B", "X" e "C" estão sobre a fronteira CCR, e assim consideradas eficientes. No caso do modelo de retornos variáveis de escala as DMUs "V", "W", "X", "Y" e "Z" formam uma fronteira convexa, envelopando as unidades consideradas ineficientes. 
Figura 1 - Modelo DEA BCC

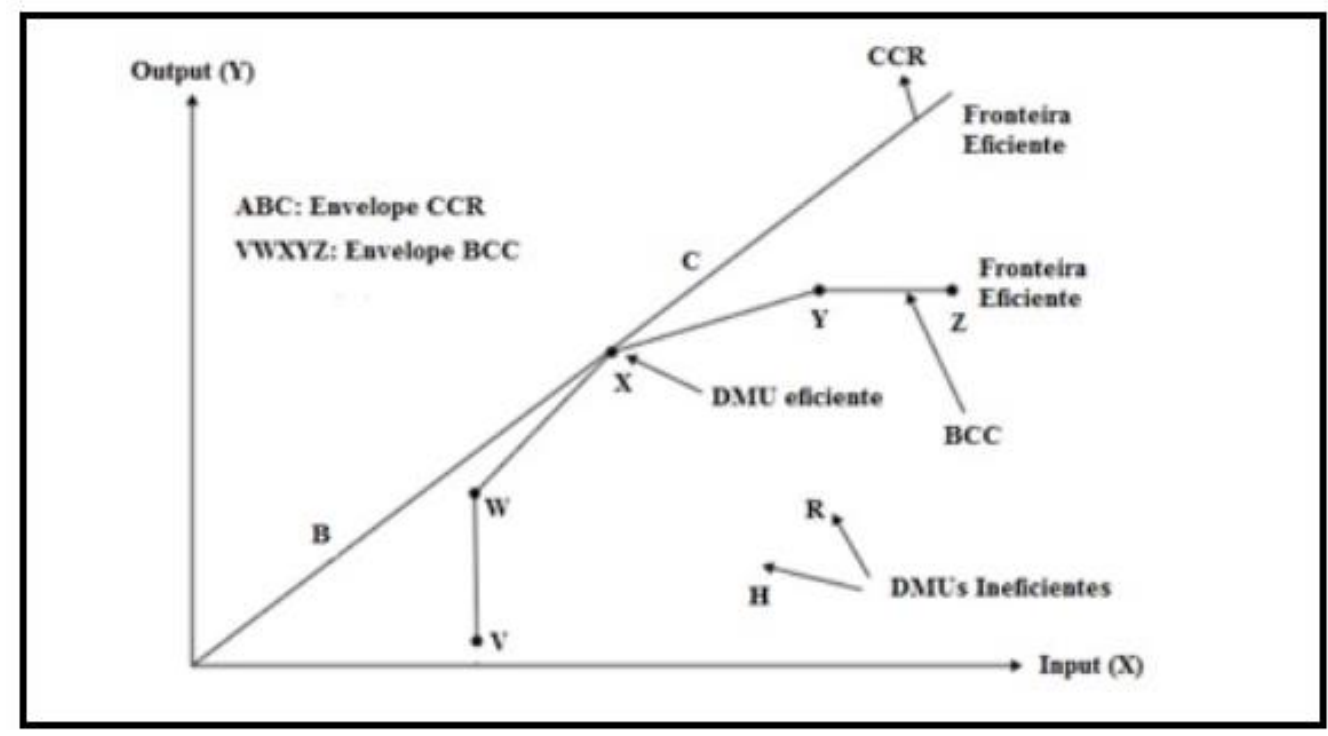

Fonte: Tavares e Meza (2017)

Portanto para esta análise foi optado por utilizar o modelo BCC, pois segundo Mello et al. (2005) substitui o axioma da proporcionalidade entre inputs e outputs pelo axioma da convexidade, ou seja, na análise das unidades, esse modelo desconsidera o tamanho, permitindo maior distribuição dos resultados independentemente do tamanho da instituição.

O modelo BCC, utiliza o retorno variável de escala (VRS), procurando, assim, evitar problemas existentes em situações de competição imperfeita. O BCC (VRS) é usado quando ocorrem Retornos Variáveis de Escala, sejam eles crescentes ou decrescentes ou mesmo constantes. A formulação do modelo BCC é representada na Figura 2.

Figura 2 - Modelo DEA BCC

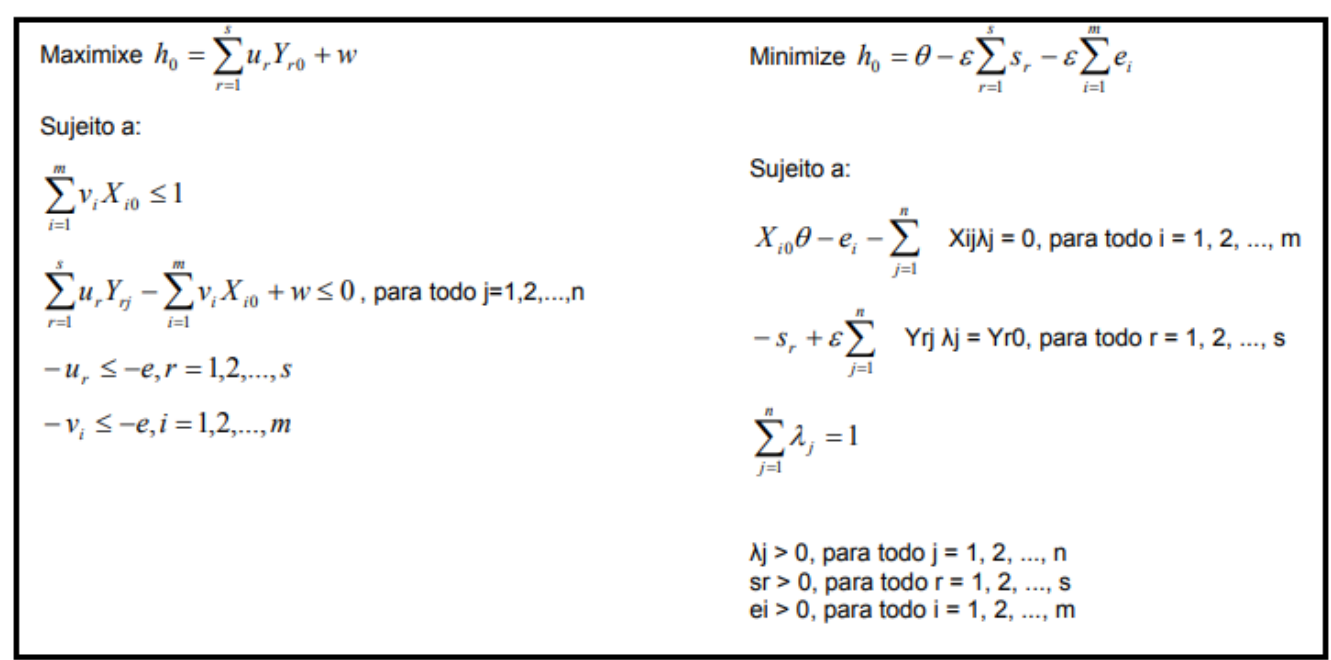

Fonte: Adaptado de Peña (2008) 
Essa técnica DEA se baseia no pressuposto de que, se uma unidade tomadora de decisão (DMU) é capaz de produzir uma determinada "saída" Y (output) utilizando X entradas (inputs), então outras unidades também poderiam fazer o mesmo, caso elas estivessem atuando eficientemente (GIACOMELLO e OLIVEIRA, 2014).

As variáveis de INPUT referem-se aos dados que se deseja diminuir e no segundo grupo encontram-se as variáveis de OUTPUT, que se referem aos dados que se deseja aumentar. Foi utilizado o modelo com orientado para OUTPUT, ou seja, os resultados priorizam as saídas, entre elas o número de alunos concluintes, o número de alunos, o numero de cursos oferecidos e a eficiência acadêmica (relação entre conclusão, evasão e retenção do ciclo de formação dos alunos). Logo, foram classificados como eficientes as instituições que, considerando o input à disposição e que conseguiram maximizar a obtenção de outputs.

O Quadro 2 demonstra os indicadores utilizados como insumos (inputs) e resultados (Outputs), e sua metodologia de cálculo.

Quadro 2 - Variáveis de Input e Output

\begin{tabular}{|c|c|}
\hline \multicolumn{2}{|c|}{ Variáveis de Input } \\
\hline Indicador & Objetivo da Plataforma \\
\hline GF & Valor gasto com Folha de pagamento na unidade de ensino. \\
\hline GM & Valor gasto com Manutenção na unidade de ensino. \\
\hline IN & Valor gasto com Investimento na unidade de ensino. \\
\hline TP & Número total de Professores na unidade de ensino. \\
\hline DM & Número de Docentes Doutores e Mestres na unidade de ensino. \\
\hline CA & Número de Campi na unidade de ensino. \\
\hline Indicador & Variáveis de Output \\
\hline CO & Número de Concluintes na unidade de ensino. \\
\hline AC & Número de alunos em curso na unidade de ensino \\
\hline CR & Número de Cursos oferecidos na unidade de ensino. \\
\hline
\end{tabular}

Fonte: dados da pesquisa

Uma maneira de fazer um ranking, conforme Angulo-Meza et al. (2005), é calculando a eficiência composta normalizada, a qual será única para cada unidade. A eficiência composta é o resultado da análise da DMU pela fronteira padrão e invertida. De acordo com Angulo-Meza et al. (2005), o resultado é obtido através da média aritmética entre a eficiência padrão e o valor obtido da subtração da eficiência invertida pela unidade, conforme Fórmula 1. 
E a eficiência composta normalizada, de acordo com Angulo-Meza et al. (2005), é obtida dividindo o valor da eficiência composta pelo maior valor entre todos os valores de eficiência composta, conforme Fórmula 2.

Eficiência Composta Normalizada = Eficiência Composta / Max (Eficiência Composta) (2)

Desta forma para fins de análise será utilizada a eficiência Composta Normalizada, que permitirá fazer uma classificação e agrupamentos das unidades do IFs pelo seu grau de eficiência.

\subsection{Coleta de Dados}

Os dados de Input e Output foram coletados na Plataforma Nilo Peçanha referente ao ano de 2018 (último ano com dados disponíveis). A plataforma Nilo Peçanha é um espaço virtual de coleta, validação e disseminação estatística oficiais da Rede Federal de Educação Profissional, Cientifica e Tecnológica (Rede Federal). Tem como objetivo reunir dados para gestão e monitoramento pela secretaria de educação profissional e tecnológica do ministério da educação. Estes dados são apresentados nas Tabelas 1 e 2.

Tabela 1 - Dados de 2018 das variáveis de Input

\begin{tabular}{|c|c|c|c|c|c|c|c|}
\hline DMU & $\begin{array}{l}\text { Gastos com } \\
\text { Folha de } \\
\text { Pagamento }\end{array}$ & $\begin{array}{l}\text { Gastos com } \\
\text { Manutenção }\end{array}$ & $\begin{array}{l}\text { Gastos com } \\
\text { Investimentos }\end{array}$ & $\begin{array}{c}\text { Profes } \\
\text { sores }\end{array}$ & $\begin{array}{l}\text { Doutores } \\
\text { Mestres }\end{array}$ & Técnicos & Campi \\
\hline UN1 & $84.800 .765,00$ & $20.351 .175,00$ & $6.472 .758,00$ & 373 & 225 & 381 & 6 \\
\hline UN2 & $326.330 .712,00$ & 70.707.241,00 & $8.257 .284,00$ & 1033 & 723 & 785 & 16 \\
\hline UN3 & $283.609 .585,00$ & $72.163 .990,00$ & $20.103 .705,00$ & 1006 & 553 & 894 & 15 \\
\hline UN4 & $68.940 .230,00$ & $24.619 .786,00$ & $8.507 .018,00$ & 312 & 157 & 323 & 6 \\
\hline UN5 & $170.828 .400,00$ & $38.554 .912,00$ & $8.916 .606,00$ & 712 & 567 & 563 & 10 \\
\hline UN6 & $452.698 .523,00$ & $104.807 .493,00$ & $9.035 .389,00$ & 1715 & 1150 & 1101 & 21 \\
\hline UN7 & $237.647 .742,00$ & $73.751 .012,00$ & $7.468 .740,00$ & 849 & 667 & 864 & 15 \\
\hline UN8 & $287.165 .990,00$ & $69.257 .683,00$ & $8.955 .507,00$ & 1038 & 884 & 864 & 15 \\
\hline UN9 & $549.498 .141,00$ & $116.966 .918,00$ & $23.675 .781,00$ & 1846 & 1442 & 1595 & 32 \\
\hline UN10 & $535.425 .699,00$ & $103.572 .964,00$ & $16.462 .937,00$ & 1637 & 1378 & 1358 & 22 \\
\hline UN11 & $323.439 .329,00$ & $71.972 .000,00$ & $13.874 .977,00$ & 1028 & 733 & 714 & 12 \\
\hline UN12 & $227.695 .928,00$ & $60.879 .464,00$ & $10.377 .798,00$ & 820 & 471 & 669 & 11 \\
\hline UN13 & $379.559 .559,00$ & $61.155 .944,00$ & $6.143 .688,00$ & 1302 & 1070 & 893 & 14 \\
\hline UN14 & $235.078 .953,00$ & $69.526 .226,00$ & $34.656 .868,00$ & 774 & 641 & 649 & 12 \\
\hline UN15 & $461.321 .318,00$ & $115.242 .519,00$ & $19.639 .418,00$ & 1796 & 1094 & 1378 & 30 \\
\hline UN16 & $324.969 .501,00$ & $65.081 .010,00$ & $15.755 .609,00$ & 1057 & 877 & 880 & 18 \\
\hline UN17 & $144.391 .125,00$ & $33.988 .828,00$ & $4.944 .232,00$ & 609 & 455 & 575 & 10 \\
\hline
\end{tabular}




\begin{tabular}{c|c|c|c|c|c|c|c}
\hline UN18 & $340.756 .244,00$ & $82.305 .121,00$ & $17.526 .795,00$ & 1232 & 839 & 845 & 19 \\
\hline UN19 & $203.409 .842,00$ & $54.869 .199,00$ & $7.096 .391,00$ & 719 & 512 & 686 & 13 \\
\hline UN20 & $383.484 .205,00$ & $76.612 .612,00$ & $23.175 .220,00$ & 1301 & 839 & 1023 & 18 \\
\hline UN21 & $430.656 .369,00$ & $78.548 .475,00$ & $24.783 .317,00$ & 1324 & 1087 & 1031 & 21 \\
\hline UN22 & $439.798 .518,00$ & $80.598 .861,00$ & $46.823 .645,00$ & 1277 & 979 & 1014 & 16 \\
\hline UN23 & $341.298 .301,00$ & $71.813 .628,00$ & $6.105 .117,00$ & 1349 & 840 & 947 & 20 \\
\hline UN24 & $300.988 .132,00$ & $66.548 .904,00$ & $21.110 .217,00$ & 1299 & 1066 & 930 & 25 \\
\hline UN25 & $346.396 .988,00$ & $57.748 .063,00$ & $15.418 .504,00$ & 1112 & 1010 & 881 & 15 \\
\hline UN26 & $477.834 .798,00$ & $103.384 .432,00$ & $20.413 .034,00$ & 1628 & 1349 & 1159 & 20 \\
\hline UN27 & $155.595 .431,00$ & $50.679 .149,00$ & $14.213 .329,00$ & 700 & 407 & 561 & 9 \\
\hline UN28 & $102.250 .804,00$ & $24.325 .604,00$ & $5.549 .872,00$ & 327 & 206 & 382 & 5 \\
\hline UN29 & $346.555 .986,00$ & $74.709 .877,00$ & $9.172 .501,00$ & 1261 & 1140 & 984 & 17 \\
\hline UN30 & $215.394 .606,00$ & $40.268 .486,00$ & $13.209 .589,00$ & 529 & 426 & 666 & 9 \\
\hline UN31 & $476.540 .107,00$ & $88.797 .471,00$ & $16.308 .278,00$ & 1605 & 1254 & 1144 & 23 \\
\hline UN32 & $143.499 .214,00$ & $39.18 .668,00$ & $22.500 .984,00$ & 495 & 324 & 530 & 7 \\
\hline UN33 & $725.753 .087,00$ & $130.160 .136,00$ & $40.622 .180,00$ & 2955 & 2412 & 1843 & 37 \\
\hline UN34 & $231.502 .932,00$ & $49.567 .915,00$ & $7.794 .870,00$ & 660 & 547 & 629 & 10 \\
\hline UN35 & $356.547 .501,00$ & $68.055 .117,00$ & $9.289 .673,00$ & 1123 & 927 & 802 & 15 \\
\hline UN36 & $208.613 .289,00$ & $56.710 .771,00$ & $14.154 .568,00$ & 618 & 552 & 553 & 9 \\
\hline UN37 & $192.831 .315,00$ & $35.614 .013,00$ & $8.218 .293,00$ & 620 & 529 & 570 & 9 \\
\hline UN38 & $176.689 .105,00$ & $54.969 .657,00$ & $10.286 .122,00$ & 721 & 510 & 584 & 11 \\
\hline
\end{tabular}

Fonte: Base de Dados Nilo Peçanha

Os dados referem-se ao ano de 2018 conforme plataforma Nilo Peçanha. As variáveis selecionadas justificam as análises da eficiência das unidades. Na Tabela 2 se mostram as variáveis Output.

Tabela 2 - Dados de 2018 das variáveis de Ouput

\begin{tabular}{c|c|c|c}
\hline DMU & Concluintes & Alunos em Curso & Cursos Oferecidos \\
\hline UN1 & 908 & 3.923 & 79 \\
\hline UN2 & 2.970 & 14.222 & 244 \\
\hline UN3 & 2.659 & 13.835 & 258 \\
\hline UN4 & 1.984 & 5.169 & 140 \\
\hline UN5 & 3.398 & 9.802 & 216 \\
\hline UN6 & 4.752 & 21.193 & 251 \\
\hline UN7 & 3.496 & 8.893 & 220 \\
\hline
\end{tabular}




\begin{tabular}{|c|c|c|c|}
\hline UN8 & 2.887 & 12.254 & 214 \\
\hline UN9 & 9.503 & 31.732 & 686 \\
\hline UN10 & 8.801 & 20.246 & 330 \\
\hline UN11 & 3.081 & 14.790 & 191 \\
\hline UN12 & 4.629 & 8.239 & 171 \\
\hline UN13 & 3.399 & 10.825 & 242 \\
\hline UN14 & 2.235 & 13.334 & 253 \\
\hline UN15 & 4.243 & 26.142 & 511 \\
\hline UN16 & 3.154 & 12.825 & 202 \\
\hline UN17 & 1.928 & 6.875 & 207 \\
\hline UN18 & 4.351 & 21.106 & 301 \\
\hline UN19 & 3.834 & 16.364 & 423 \\
\hline UN20 & 4.431 & 13.981 & 497 \\
\hline UN21 & 2.377 & 21.670 & 242 \\
\hline UN22 & 5.079 & 17.576 & 283 \\
\hline UN23 & 4.293 & 16.907 & 353 \\
\hline UN24 & 5.382 & 21.628 & 349 \\
\hline UN25 & 2.838 & 10.905 & 151 \\
\hline UN26 & 11.864 & 24.598 & 533 \\
\hline UN27 & 2.916 & 12.903 & 175 \\
\hline UN28 & 1.543 & 3.653 & 92 \\
\hline UN29 & 7.699 & 15.728 & 307 \\
\hline UN30 & 1.469 & 5.871 & 103 \\
\hline UN31 & 10.522 & 20.519 & 721 \\
\hline UN32 & 2.558 & 5.592 & 201 \\
\hline UN33 & 16.014 & 30.694 & 854 \\
\hline UN34 & 3.080 & 9.150 & 211 \\
\hline UN35 & 4.031 & 17.373 & 251 \\
\hline UN36 & 7.746 & 10.933 & 263 \\
\hline UN37 & 2.706 & 7.050 & 183 \\
\hline UN38 & 2.197 & 8.622 & 147 \\
\hline
\end{tabular}

Fonte: Base de Dados Nilo Peçanha

A partir das variáveis apresentadas no Quadro 2, os dados da Tabela 1 foram tabulados em planilha eletrônica Microsoft Excel ${ }^{\circledR}$ e posteriormente analisado utiliza pelo sistema integrado de apoio a decisão SIAD v3.0, desenvolvido por MEZA et al. (2005). 


\section{RESULTADOS E DISCUSSÃO}

Os dados foram analisados com base nos oito indicadores da base de dados Nilo Peçanha, que são dadas as variáveis de inputs e outputs utilizadas na Análise Envoltória de Dados. Todas as unidades selecionadas continham as informações para serem calculadas. As IFs da amostra do estudo somaram 38 unidades espalhas pelo Brasil.

Os resultados são apresentados na Tabela 3. O modelo BCC utilizado é menos restritivo que o CCR, pois a DMU eficiente no modelo CCR será também eficiente no modelo BCC, porém o contrário não é verdadeiro (FERREIRA; BRAGA, 2007).

Observa-se na Tabela 3 a saída do resultado feito pelo software DEAP, com os cálculos das eficiências técnicas para cada unidade dos Institutos Federais. Os resultados apresentam as IFs que estão na fronteira de eficiência, ou seja, são as que possuem eficiência técnica igual a 1,0. As IFs ineficientes também aparecem nessa tabela com eficiência técnica menor que 1,0 .

Tabela 3 - Resultado da Eficiência dos IFs

\begin{tabular}{c|c|c|c}
\hline DMU & $\begin{array}{c}\text { Eficiência } \\
\text { Técnica }\end{array}$ & \multicolumn{1}{c}{ Benchmark } & $\begin{array}{c}\text { Composta } \\
\text { Normalizada* }\end{array}$ \\
\hline UN1 & 1,00 & - & 0,70 \\
\hline UN2 & 0,80 & UN6, UN9, UN 19 & 0,56 \\
\hline UN3 & 0,81 & UM 15, UN 19 & 0,57 \\
\hline UN4 & 1,00 & - & 0,70 \\
\hline UN5 & 0,88 & UN 4, UN 17, UN 19, & 0,82 \\
\hline UN6 & 1,00 & UN 20, UN 27, UN 28 & 0,70 \\
\hline UN7 & 0,60 & UN 17, UN 19, UN 20, & 0,42 \\
\hline UN8 & 0,68 & UN 6, UN 19, UN 26 & 0,47 \\
\hline UN9 & 1,00 & - & 0,70 \\
\hline UN10 & 0,86 & UN 6, UN 9, UN 19, & 0,60 \\
\hline UN11 & 0,93 & UN 19, UN 26, UN 27 & 0,67 \\
\hline UN12 & 0,64 & UN 19, UN 20, UN 27, & 0,44 \\
\hline UN13 & 0,87 & UN 28, UN 36 & 0,46 \\
\hline UN14 & 0,87 & UN 18, UN 19, UN 20, & 0,61 \\
\hline UN15 & 1,00 & UN 23 27 & 0,60 \\
\hline UN16 & 0,66 & UN 9, UN 19, UN 24 & 0,70 \\
\hline UN17 & 1,00 & - & 0,74 \\
\hline & & & - \\
\hline
\end{tabular}




\begin{tabular}{l|l|c|l}
\hline UN18 & 1,00 & - & 0,83 \\
\hline UN19 & 1,00 & - & 1,00 \\
\hline UN20 & 1,00 & - & 0,70 \\
\hline UN21 & 0,97 & UN 9, UN 18, UN 19 & 0,68 \\
\hline UN22 & 0,87 & UN 19, UN 26, UN 27 & 0,61 \\
\hline UN23 & 1,00 & - & 0,74 \\
\hline UN24 & 1,00 & - & 0,70 \\
\hline UN25 & 0,63 & UN 9, UN 19, UN 24 & 0,44 \\
\hline UN26 & 1,00 & - & 0,81 \\
\hline UN27 & 1,00 & - & 0,87 \\
\hline UN28 & 1,00 & - & 0,70 \\
\hline UN29 & 0,91 & UN 6, UN 19, UN 20, & 0,71 \\
\hline UN30 & 0,56 & UN 23 & 0,39 \\
\hline UN31 & 1,00 & UN 4, UN 19 & 0,74 \\
\hline UN32 & 1,00 & - & 0,70 \\
\hline UN33 & 1,00 & - & 0,70 \\
\hline UN34 & 0,82 & UN 6, UN 19, UN 20, & 0,73 \\
\hline UN35 & 0,96 & UN 6, UN 9, UN 19, & 0,83 \\
\hline UN36 & 1,00 & UN 26 & - \\
\hline UN37 & 0,74 & UN 4, UN 19, UN 20, & \\
\hline & & UN 4, UN 19, UN 27 & - \\
\hline
\end{tabular}

* Eficiência Composto Normalizada

Fonte: Dados da Pesquisa

O benchmark das unidades ineficientes é determinado pela projeção destas na fronteira de eficiência. A forma como é feita esta projeção determina a orientação do modelo: orientação a inputs, quando a eficiência é atingida por uma redução equiproporcional de entradas, mantidas as saídas constantes; e orientação a outputs, quando se deseja maximizar os resultados sem diminuir os recursos. (MELO et al., 2005).

Em 2018, na Tabela 3 demonstra que foram encontradas 18 IFs consideradas eficientes, representando $47,36 \%$ das IFs que estão na fronteira de eficiência. Sendo que 14 dessas IFES serviram de referência (benchmark) para as outras IFs ineficientes, de acordo com os resultados do DEAP. As 14 IFs são, por ordem decrescente de quantidades de vezes que serviram de referências: o IFNMG- UN 19 (20 vezes), o IFPA-UN20 (8 vezes), o IFROUN27 ( 7 vezes), IFBA-UN 6 e IFCE-UN 9 (6 vezes), IFRN-UN 26 (5 vezes), IFAP-UN 4 e IFRR-UN 28 (4 vezes), IFPI-UN 23 e IFMS-UN 17 (3 vezes), IFMT-UN 18 e IFPR-24 (2 
vezes) e IFMA-UN 15 (1 vez). Isso mostra o grau de importância dessas IFs em relação às que são eficientes.

Quanto às 20 IFs que não são eficientes e, que representam $52 \%$ da amostra, podemos destacar as seis mais ineficientes (valores de eficiência abaixo de um) que são a IFMG-UN 16 $(0,66)$, IFRARROUPILHA-UN $12(0,64)$, IFRJ-UN $25(0,63)$, IFBAIANO-UN $7(0,60)$ e o IFS-UN $30(0,56)$.

Não foi possível fazer comparação com outros trabalhos sobre eficiência dos IFs, pois não foi encontrado pesquisas com os IFs de outros períodos. Realizando a análise da Eficiência Composta Normalizada, foi possível notar que a primeira das instituições altamente eficiente é a UN 19 IFNMG, ou seja, o Instituo Federal do Norte de Minas Gerais. Após estes resultados, buscou analisar as unidades quanto à eficiência acadêmica de cada unidade e sua relação à eficiência técnica.

A Eficiência acadêmica é deve avaliar a capacidade da Rede Federal de atingir os resultados previstos em termos de "estudantes certificados" ou "com potencial de certificação" em relação à quantidade total de matrículas considerando um determinado ciclo de matrículas. Assim como ocorre nos demais "Indicadores de Ciclo" a análise da Eficiência Acadêmica será realizada considerando a situação de matrícula dos alunos com fím de ciclo previsto para o ano anterior ao ano de referência a partir da medição em 31/12 do ano em referencia.

Este indicador mede o percentual de alunos que concluíram o curso com êxito dentro do período previsto (+ 1 ano), acrescido de um percentual (projeção) dos alunos retidos no ano de referência que poderão concluir o curso. São considerados apenas os alunos matriculados em ciclos de matrícula com término previsto para o ano anterior ao Ano de Referência. Para este cálculo é empregado o conceito de matrícula e não de matrícula equivalente. A Meta prevista para este indicador é derivada da meta de conclusão contida na estratégia 11.11 e 12.3 da Lei 13.005/2014.

$\mathrm{Na}$ Tabela 4, comparando-se à eficiência técnica em relação a eficiência acadêmica composta pela base Nilo Peçanha.

Tabela 4 - Resultado da Eficiência Técnica dos IFs e Eficiência Acadêmica

\begin{tabular}{c|c|c|c}
\hline $\begin{array}{c}\text { Classificação } \\
\text { da Eficiência } \\
\text { Acadêmica }\end{array}$ & $\begin{array}{c}\text { Indicador da } \\
\text { Eficiência } \\
\text { Acadêmica }\end{array}$ & DMU & $\begin{array}{c}\text { Indicador da Eficiência } \\
\text { Técnica }\end{array}$ \\
\hline $\mathbf{1}^{\mathbf{0}}$ & $\mathbf{7 3 , 6}$ & UN 4 & $\mathbf{1 , 0 0}$ \\
\hline $\mathbf{2}^{\mathbf{o}}$ & $\mathbf{7 1 , 2}$ & UN 27 & $\mathbf{1 , 0 0}$ \\
\hline $\mathbf{3}^{\mathbf{0}}$ & $\mathbf{6 2 , 8}$ & UN 26 & $\mathbf{1 , 0 0}$ \\
\hline $4^{\mathbf{0}}$ & 59,2 & UN 29 & 0,91 \\
\hline $\mathbf{5}^{\mathbf{0}}$ & $\mathbf{5 7 , 4}$ & UN 15 & $\mathbf{1 , 0 0}$ \\
\hline $6^{\mathbf{0}}$ & 56,0 & UN 16 & 0,66 \\
\hline $7^{\mathbf{0}}$ & 54,5 & UN 10 & 0,86 \\
\hline $\mathbf{8}^{\mathbf{0}}$ & $\mathbf{5 4 , 5}$ & UN 18 & $\mathbf{1 , 0 0}$ \\
\hline $9^{\mathbf{o}}$ & 54,1 & UN 7 & 0,60 \\
\hline
\end{tabular}




\begin{tabular}{|c|c|c|c|}
\hline $10^{\circ}$ & 54,0 & UN 35 & 0,96 \\
\hline $11^{\circ}$ & 53,8 & UN 8 & 0,68 \\
\hline $12^{\circ}$ & 51,1 & UN 28 & 1,00 \\
\hline $13^{\circ}$ & 51,0 & UN 33 & 1,00 \\
\hline $14^{\circ}$ & 49,3 & UN 12 & 0,64 \\
\hline $15^{\circ}$ & 48,9 & UN 3 & 0,81 \\
\hline $16^{\circ}$ & 47,9 & UN 9 & 1,00 \\
\hline $17^{\circ}$ & 47,2 & UN 13 & 0,87 \\
\hline $18^{\circ}$ & 47,0 & UN 20 & 1,00 \\
\hline $19^{\circ}$ & 46,8 & UN 32 & 1,00 \\
\hline $20^{\circ}$ & 46,8 & UN 37 & 0,74 \\
\hline $21^{\circ}$ & 46,7 & UN 31 & 1,00 \\
\hline $22^{\circ}$ & 46,0 & UN 2 & 0,80 \\
\hline $23^{\circ}$ & 45,8 & UN 6 & 1,00 \\
\hline $24^{\circ}$ & 45,4 & UN 1 & $\overline{1,00}$ \\
\hline $25^{\circ}$ & 45,2 & UN 23 & 1,00 \\
\hline $26^{\circ}$ & 45,0 & UN 30 & 0,56 \\
\hline $27^{\circ}$ & 44,6 & UN 34 & 0,82 \\
\hline $28^{\circ}$ & 44,5 & UN 14 & 0,87 \\
\hline $29^{\circ}$ & 44,0 & UN 24 & 1,00 \\
\hline $30^{\circ}$ & 44,0 & UN 38 & 0,65 \\
\hline $31^{\circ}$ & 43,7 & UN 22 & 0,87 \\
\hline $32^{\circ}$ & 43,4 & UN 19 & 1,00 \\
\hline $33^{\circ}$ & 41,7 & UN 11 & 0,93 \\
\hline $34^{\circ}$ & 40,3 & UN 25 & 0,63 \\
\hline $35^{\circ}$ & 37,4 & UN 17 & 1,00 \\
\hline $36^{\circ}$ & 37,2 & UN 5 & 0,88 \\
\hline $37^{\circ}$ & 32,8 & UN 36 & 1,00 \\
\hline $38^{\circ}$ & 28,8 & UN 21 & 0,97 \\
\hline
\end{tabular}

Fonte: Dados da Pesquisa.

Os resultados demonstram que em 2018, conforme Tabela 4, entre os IFs consideradas eficientes na análise DEA, nem todos apresentam Eficiência Acadêmica esperada. Considerando os 10 primeiros IFs com melhor Eficiência Acadêmica, somente os IFAP-UN 4, IFRO UN-27, IFRN UN-26, IFMA UN-15, IFMT UN-18 estão classificadas como eficientes quanto à análise pelo DEA. 
Pode se notar também as IFSULDEMINAS UN-36, IFMS UN-17, IFNMG UN-19 e IFPR UN-24 estão entre as 10 últimas considerada a Eficiência Acadêmica, porém estas foram consideradas eficientes tecnicamente pelo DEA. Importante destacar que o IFNMG foi o mais utilizado no Banchmark e mesmo assim não esta entre os melhore em relação à Eficiência Acadêmica.

Isso demonstra que mesmo com eficiência técnica (DEA) não se pode garantir que os alunos conseguem terminar o ciclo esperada com base nos objetivos da Lei 13.005/2014, ou seja, a eficiência acadêmica. Estes objetivos compreendem elevar gradualmente a taxa de conclusão média dos cursos técnicos de nível médio na Rede Federal de Educação Profissional, Científica e Tecnológica para 90\% (noventa por cento) e elevar, nos cursos presenciais, a relação de alunos (as) por professor para 20 (vinte) e elevar gradualmente a taxa de conclusão média dos cursos de graduação presenciais nas universidades públicas para $90 \%$ (noventa por cento), ofertar, no mínimo, um terço das vagas em cursos noturnos e elevar a relação de estudantes por professor (a) para 18 (dezoito), mediante estratégias de aproveitamento de créditos e inovações acadêmicas que valorizem a aquisição de competências de nível superior.

\section{CONSIDERAÇÕES FINAIS}

Nessa pesquisa buscou-se avaliar o desempenho das IFs quanto à eficiência técnica em relação aos gastos de recursos públicos e estrutura oferecida a cada unidade em 2018 e verificar a relação com capacidade da Eficiência Acadêmica.

De acordo com os resultados da pesquisa, verificou-se que houve a validação do modelo ajustado que considerava a relação das IFs eficientes e não eficientes, utilizando os insumos e produtos estabelecidos, com as variáveis ambientais selecionadas.

A gestão de recursos públicos implica uma expectativa que o investimento realizado após a criação dos IFs ganhos de eficiência quanto à formação e capacitação da população gerando maior competitividade interna e externa, gerando competências profissionais através dos cursos técnicos e desenvolvimentos regionais. Porém o que se observou em 2018 foi que menos da metade (47\%) das IFs são consideradas eficientes.

Entre as unidades consideradas eficientes no ano de 2018 estão os IFs o IFNMG, o IFPA, o IFRO, IFBA, IFCE, IFRN, IFAP e IFRR, IFPI e IFMS, IFMT e IFPR e IFMA. Furtado (2015) avaliou a eficiência dos IFs em 2012 a 2013 e apresentou também a eficiência dos IFRR, IFMA, IFRO com dados extras.

Outro ponto importante dos resultados: notou-se que nem todos os IFs que foram considerados eficientes tecnicamente estão classificados como Eficientes Acadêmicos. A Meta prevista com Eficiência Acadêmica é derivada da meta de conclusão contida na estratégia 11.11 e 12.3 da Lei 13.005/2.014 do Plano Nacional da Educação e pelos resultados mostrou que algumas instituições, mesmo ineficientes tecnicamente estão conseguindo cumprir as metas acadêmicas estabelecidas.

Sugere-se que pesquisas futuras utilizem novas variáveis de insumos e produtos, assim como outras variáveis ambientais que possam corroborar com os testes aplicados nesse estudo. Ou aplicar em anos seguintes, com o intuito de testar em relação ao tempo, se as unidades vêm evoluindo quanto sua eficiência. 


\section{REFERÊNCIAS}

ALMEIDA, A. T. C.; ALMEIDA FILHO, Á. C. Eficiência técnica da gestão das escolas federais de educação básica no Brasil. Ciências Sociais em Perspectiva, Cascavel, v. 13, n. $25,2014$.

AOKI, S.; INOUE, K.; GEJIMA, R. Data envelopment analysis for evaluating Japanese universities. Artificial Life and Robotics, v.15, p. 165-170, 2010. DOI:10.1007/s10015010-0786-7

COSTA, E. M.; RAMOS, F. S.; SOUZA, H. R.; SAMPAIO, L. M. B.; BARBOSA, R. B. Dinâmica da eficiência produtiva das instituições federais de ensino superior. 2015. Planejamento e Políticas Públicas, n. 44, p. 51-54, 2015.

CORBUCCI, P. R. As universidades federais: gasto, desempenho, eficiência e produtividade. Brasília: IPEA, Texto para discussão, n. 752, 2000

DEBREU, G. The coeficiente of resource utilization. Econometrica: Journal of the Econometric Society, v.19, n. 3, p. 273-292, 1951. Disponível em: http://www.jstor.org/stable/1906814?seq=1\#page_scan_tab_contents.

FURTADO, L. L.; CAMPOS, G. M. Grau de eficiência técnica dos Institutos Federais de Educação, Ciência e Tecnologia e a relação dos custos, indicativos de expansão e retenção junto aos escores de eficiência. Revista de Educação e Pesquisa em Contabilidade, v. 9, n. $3,2015$.

FARREL, M.J.The measurement of produtive efficiency.Journal of Royal Statistical Society, v. 120, n.3, p.253-290, 1957.Disponívelem: http://www.jstor.org/stable/2343100

FERREIRA, M. A. M.; BRAGA, M. J. Eficiência das sociedades cooperativas e de capital na indústria de laticínios. Revista Brasileira de Economia, v. 61, n. 2, p. 231-244, 2007.

GIACOMELLO, C. P.; OLIVEIRA, R. L. de. Análise Envoltória de Dados (DEA): uma proposta para avaliação de desempenho de unidades acadêmicas de uma universidade. Revista Gestão Universitária na América Latina - GUAL, Florianópolis, v. 7, n. 2, p. 130-151, maio 2014. DOI: 10.5007/1983-4535.2014v7n2p130.

KUAH, C. T.; WONG, K. Y. Efficiency assessment of universities through data envelopment analysis. Procedia Computer Science, v. 3, p. 499-506, 2011. DOI:10.1016/ j.procs.2010.12.084

KOOPMANS, T. C. Efficientallocationofresources. Econometrica: JournaloftheEconometric Society,v. 19, n.1, p. 455-465, 1951. Disponível em: http://www.jstor.org/stable/1907467?seq=1\#page_scan_tab_contents.

MELlO, J. C. C. B. S. de; MEZA, L. Â.; GOMES, E. G.; BIONDI NETO, L. Curso de análise de envoltória de dados. Simpósio Brasileiro de Pesquisa Operacional, v. 37, p. 2521-2547, 2005.

MEZA, L. A.; BIONDI NETO, L.; MELlO, J. C. C. B. S. de; GOMES, E. G.: COELHO, P.H.G. Free software for decision analysis: a software package for data envelopment models. In: 7th International Conference on Enterprise Information Systems - ICEIS 2005, v. 2, p. 207-212,2005.

PEÑA, C. R. Um Modelo de Avaliação da Eficiência da Administração Pública através do Método Análise Envoltória de Dados (DEA). Revista de Administração Contemporânea, v. 12, n. 1, p. 83-106, 2008. DOI:10.1590/S1415-65552008 000100005. 
RAMOS, R. E.; FERREIRA, G. M. Analisando retornos de escala usando DEA: um estudo em Instituições de Ensino Tecnológico no Brasil. Gepros: Gestão da Produção, Operações e Sistemas, v. 2, n. 4, 2007.

RODRIGUES, Alexandre de Cassio; MUYLDER, Cristiana Fernandes de; GONTIJO, Tiago Silveira. Eficiencia das Undiades do CEFET-MG: Uma avaliação por Data Envelopment Analysis. Revista For Science, 2018. DOI: 10.29069/forscience.2018v6n3.e270

SANTOS, J. P. P. dos. Análise da Eficiência de Unidade de Ensino: um estudo comparativo da evolução do desempenho entre os campi do Instituo Federal Farroupilha. Dissertação do Programa de Pós Graduação Profissional em Gestão de Organizações Públicas da Universidade Federal de Santa Maria, 2018.

SILVA, Juliana Sales; CORRÊA, Carolina Rodrigues; GOMES, Adriano Provezano. Determinantes da eficiência dos programas de pós-graduação em economia do Brasil. Reflexões Econômicas, v. 2, n. 2, p. 55-75, 2017.

TAVARES, R. S.; MEZA, L. A. Uso da análise envoltória de dados para a avaliação da eficiência em cursos de graduação: um estudo de caso em uma Instituição de Ensino Superior brasileira. Revista Espacios, v. 38, n. 20, 2017. 